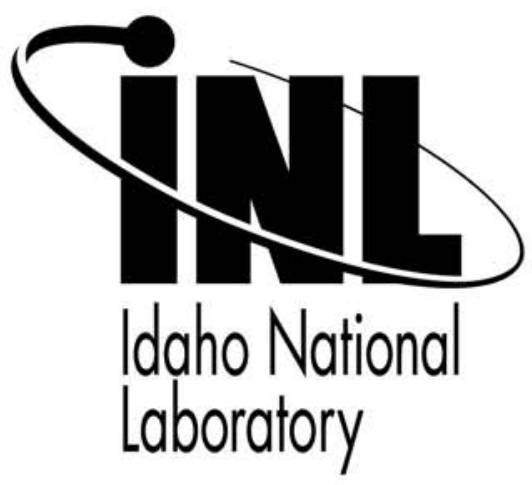

\section{IEEE Nuclear Science Symposium}

\author{
W. Scates \\ J.K. Hartwell \\ R. Aryaeinejad \\ M.E. Mcllwain \\ October 2005
}

This is a preprint of a paper intended for publication in a journal or proceedings. Since changes may not be made before publication, this preprint should not be cited or reproduced without permission of the author. This document was prepared as an account of work sponsored by an agency of the United States Government. Neither the United States Government nor any agency thereof, or any of their employees, makes any warranty, expressed or implied, or assumes any legal liability or responsibility for any third party's use, or the results of such use, of any information, apparatus, product or process disclosed in this report, or represents that its use by such third party would not infringe privately owned rights. The views expressed in this paper are not necessarily those of the United States Government or the sponsoring agency. 


\title{
Optimization Studies of a Compton Suppression Spectrometer Using Experimentally Validated Monte Carlo Simulations
}

\author{
W. Scates, J.K. Hartwell, R. Aryaeinejad, M.E. McIlwain \\ Global Technologies Incorporated, Idaho Falls, Idaho 83415 \\ Idaho National Laboratory, Idaho Falls, Idaho 83415
}

\begin{abstract}
Recent developments associated with room temperature semiconductor detectors and inorganic scintillators suggest that these detectors may be viable alternatives for the primary detector in a Compton Suppression Spectrometer (CSS). The room temperature operation of these detectors allows removal of a substantial amount of material from between primary and secondary detector and, if properly designed should afford substantially better suppression factors than can be achieved by germanium-based spectrometers. We have chosen to study the optimum properties of a CSS with a $\mathrm{LaX}_{3}: \mathrm{Ce}$ scintillator (where $X$ is chloride or bromide) as the primary gamma ray detector. A Monte Carlo photon transport model is used to determine the optimum geometric properties of this spectrometer. To validate the assumptions and basic design of the Monte Carlo simulations, the energy distribution of a ${ }^{137} \mathrm{Cs}$ point source is measured and simulated for two experimental systems. Comparison of the suppression factors for the measured and simulated data validates the model accuracy. A range of CSS physical parameters are studied to determine optimal detector geometry and to maximize the Compton suppression factor. These physical parameters and their optimum values are discussed.
\end{abstract}

\section{INTRODUCTION}

Compton-suppression systems (CSS) are routinely used in a range of research and analysis applications [1,2,3]. Commonly, these units incorporate high-purity germanium detectors. Ce-doped lanthanum halides scintillators $\left(\mathrm{LaX}_{3}: \mathrm{Ce}\right)$ provide adequate density, speed, and high light output [11]. The high photon yields translate directly into reasonable energy resolution (2.5 to $4.0 \%$ ) [4,5]. NEED to fix references

Monte Carlo simulations are a convenient tool to predict the suppression factor, to evaluate the properties of a CSS and to trend a system's behavior relative to one or more parameters. The results obtained from these modeling exercises can be used to design an actual CSS.

\section{A. Experimental Approach}

In this paper, we present MCNP transport calculations for a range of CSS design parameters. Prior MCNP simulations show that the germanium CSS suppression factor strongly depends on the location of the primary detector (PD) within the suppression detector (SD) [6], on the radial width of the $\mathrm{SD}[7]$, and on the orientation of the $\mathrm{PD}[8]$. We have determined similar trends for the Compton suppression factors of $\mathrm{LaX}_{3}: \mathrm{Ce}$ detectors. The results obtained from these modeling exercises can be used to design an actual CSS.

\section{B. Experimental Validation}

We have modeled the performance of two CSS that we have in our laboratory. The first CSS is depicted in Figure 1a. The PD is a $\varnothing 12.5 \mathrm{~mm}$ by $15 \mathrm{~mm} \mathrm{LaCl} 3: 10 \% \mathrm{Ce}$. The PD is enclosed inside an aluminum case having a thickness of 0.5 $\mathrm{mm}$ and an additional $0.4 \mathrm{~mm}$ of aluminum that lines the walls of the wells. The PD and associated light pipe are positioned in the center of a $\varnothing 76$ by $76 \mathrm{~mm} \mathrm{NaI(Tl)} \mathrm{secondary} \mathrm{detector.}$

The second CSS is depicted in figure $1 \mathrm{~b}$. The secondary detector was fabricated from BC-408 plastic scintillator. The PD was a $\varnothing 13 \mathrm{~mm}$ by $13 \mathrm{~mm} \mathrm{LaCl}_{3}: 10 \%$ Ce crystal enclosed within aluminum can. The cubic plastic scintillator (sized 200 $\mathrm{mm}$ by $200 \mathrm{~mm}$ by $200 \mathrm{~mm}$ ) equipped with centerline and transverse wells formed the SD. The PD is inside the transverse well and is located $75 \mathrm{~mm}$ from the front surface of the SD. A ${ }^{137}$ Cs point source was located $100 \mathrm{~mm}$ from the center of the detector.

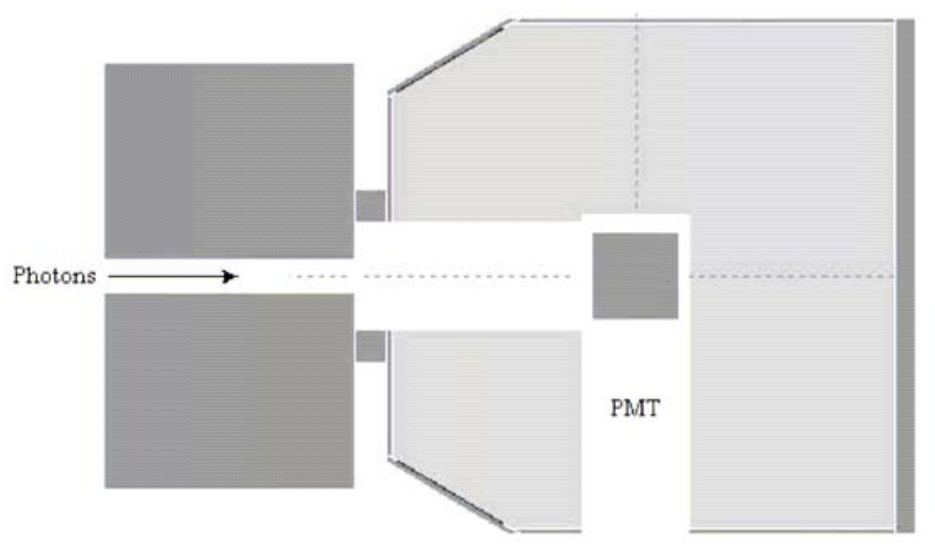

Figure 1a: Modeled representation of the first Compton suppression system. The PD is a $\varnothing 12.5 \mathrm{~mm}$ by $15 \mathrm{~mm} \mathrm{LaCl}_{3}: 10 \% \mathrm{Ce}$. 


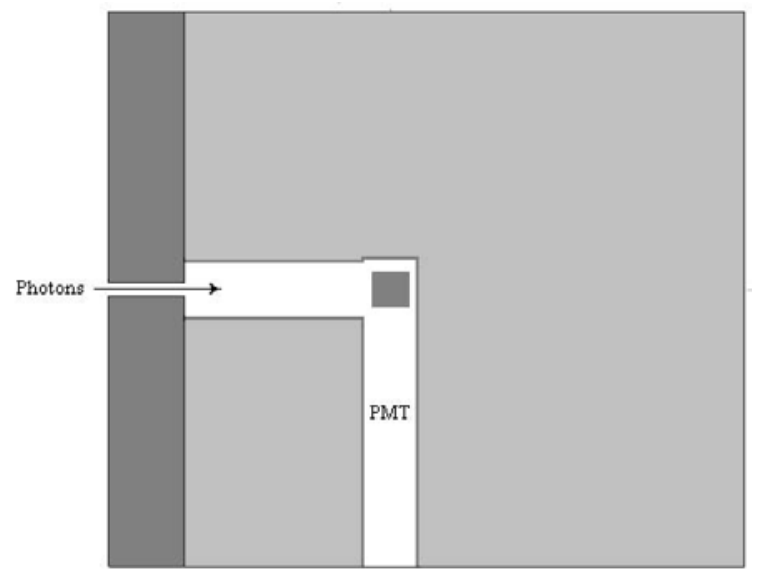

Figure 1b: Second modeled representation of the Compton Suppression System. This CSS has a $\varnothing 13 \mathrm{~mm}$ by $13 \mathrm{~mm} \mathrm{LaCl}_{3}: 10 \%$ Ce crystal enclosed within aluminum can as the $\mathrm{PD}$.

CSS systems similar to those modeled in Figure $1 \mathrm{a}$ and $1 \mathrm{~b}$ were assembled in our laboratory. For the first CSS a single photomultipler positioned perpendicular to the centerline well detected the PD light. A single PMT aligned parallel to the centerline well was employed to detect the SD signal in the NaI secondary.

The secondary BC-408 detector of the second CSS was viewed by two $50.8 \mathrm{~mm}$ diameter PMTs. The PD was monitored using a R4277 photomultipler (PMT) and a incorporated a $\varnothing 20 \mathrm{~mm} \times 50 \mathrm{~mm}$ quartz light pipe between the scintillator crystal and PMT.

Spectra were acquired both with and without suppression on both of the CSS units. $\mathrm{A}^{137} \mathrm{Cs}$ source was positioned $100 \mathrm{~mm}$ from the center of the PD and carefully aligned with the photon inlet aperture of the SD (see Figures 1a and 1b). The spectra were analyzed to determine the Compton suppression factor of each configuration. The suppression factor used for the laboratory measurements is calculated as:

$$
\text { Suppression Factor }=\frac{\text { Peak }- \text { to }- \text { Compton ratio with } \sup \text { pression }}{\text { Peak }- \text { to }- \text { Compton ratio without } \sup \text { pression }}
$$

where the peak-to-Compton ratio is defined as the ratio of the number of counts in the highest photopeak channel to the average counts in the associated Compton continuum region (358 to $382 \mathrm{keV}$ ).

\section{The Monte Carlo simulation}

The Monte Carlo simulation was performed using MCNPX Version 2.5.E [9] with standard material libraries. To calculate the suppression anticoincidence tallies were used with a minimum deposited energy in the SD for cancellation of $10 \mathrm{keV}$.

The ${ }^{137} \mathrm{Cs}$ photopeak Compton continuum suppression factor is calculated from the MCNP results as:
Suppression Factor $=\frac{P(358-382) N S}{P(358-382) S}$

Where $\mathrm{P}(358-382) \mathrm{NS}$ is the probability of events in the 358 $\mathrm{keV}$ to $382 \mathrm{keV}$ energy range without suppression and $\mathrm{P}(358$ 382)S is the probability of events with suppression.

\section{Model Validation}

To validate the MCNP detector model, predicted energy distributions for a specific activity ${ }^{137} \mathrm{Cs}$ point source were modeled for each of the above detectors. These detected photon energy distributions and the corresponding Compton suppression factors were compared to experimental data for the two CSS detectors. Figure 2 displays experimental data in comparison with model predictions. The Monte Carlo prediction energy spectrum was processed using a Gaussian smearing function to simulate the energy resolution of the scintillator.

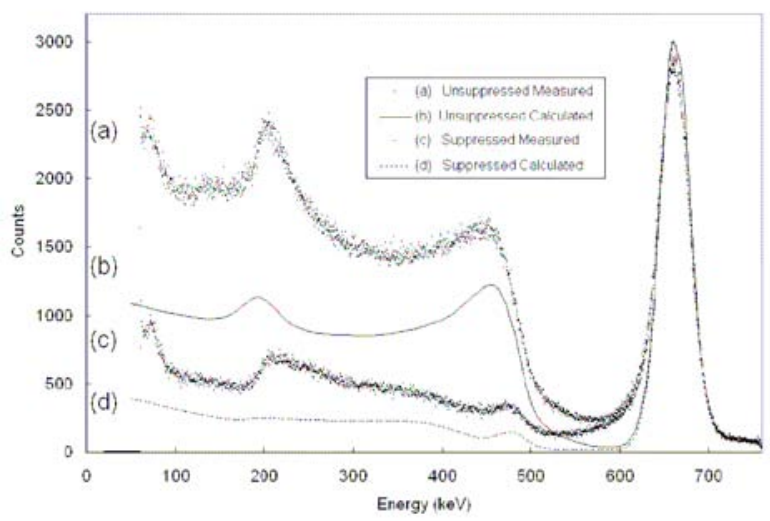

Figure 2: Experimental data compared with model predictions.

Table 1 compares the corresponding suppression factors for these two detector configurations.

TABLE I

COMPARISON OF MEASURED VERSUS MODELED COMPTON SUPPRESSION FACTORS FOR THE TWO CSS UNITS

\begin{tabular}{|l|c|c|}
\hline & $\begin{array}{c}\mathrm{NaI}(\mathrm{Tl}) \\
\text { Detector }\end{array}$ & $\begin{array}{c}\text { Plastic } \\
\text { Scintillator } \\
\text { Detector }\end{array}$ \\
\hline Model & 4.1 & 2.55 \\
\hline Experimental & 3.5 & 2.57 \\
\hline
\end{tabular}

The calculations using MCNP match the measured suppression factor reasonably well at energies below $400 \mathrm{keV}$ as is shown in Figure 3. However, in the energy regime from 400 to $550 \mathrm{keV}$ the calculated suppression factor deviates from the measured values by as much as a factor of two. These events correspond to both high angle scatters and multiscatter events in the detector. 


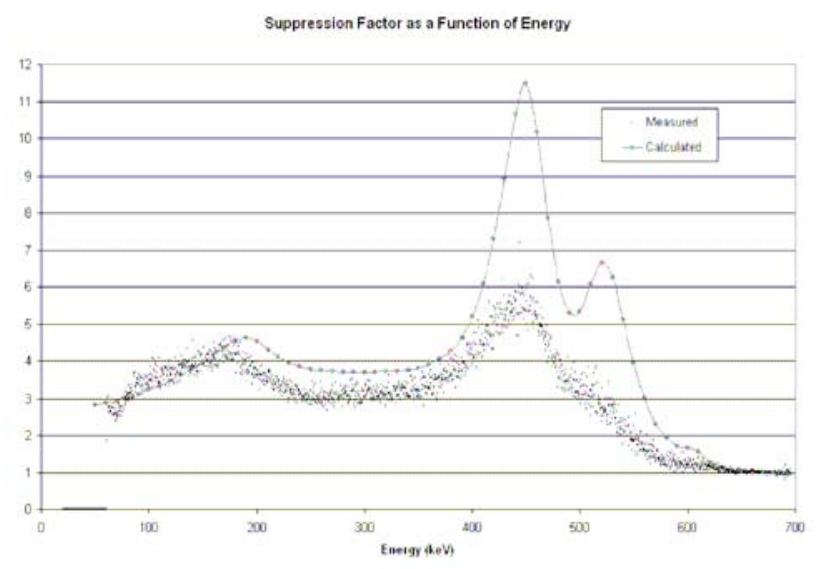

Figure 3: the resulting suppression factors determined as a function of energy. For most energy values, the model and measurements provide approximately the same suppression factor, but differ strongly in the 400 to $600 \mathrm{keV}$ region.

It is believed that the discrepancy above $400 \mathrm{keV}$ comes from the dead materials lining the inner walls of the SD being to thin in the calculation. This causes a two-pronged effect on the suppression factor in this range. First, the inner diameter of the active SD materials would increase which directly reduces the suppression factor of high angle scatters. Second, the increase in dead material increases the attenuation of the photons exiting the primary detector.

Calculations with increased dead layer thickness better approximated the experimental data. However, only results using the initial geometrical parameters are shown because, in general, we did not want to adjust the calculation geometry to specifically match this experimental data. These results provide sufficient justification to validate the use of this MCNP model to predict optimal CSS detector properties.

\section{E. CSS Optimization}

The simplified detector geometry that was used for the parameter optimization study is shown in Figure 4. The PD was the $\varnothing 13 \mathrm{~mm}$ by $13 \mathrm{~mm} \mathrm{LaCl}_{3}$ :Ce detector modeled in the validation study. The $\mathrm{SD}$ was $\mathrm{NaI}(\mathrm{Tl})$ with cylindrical symmetry.

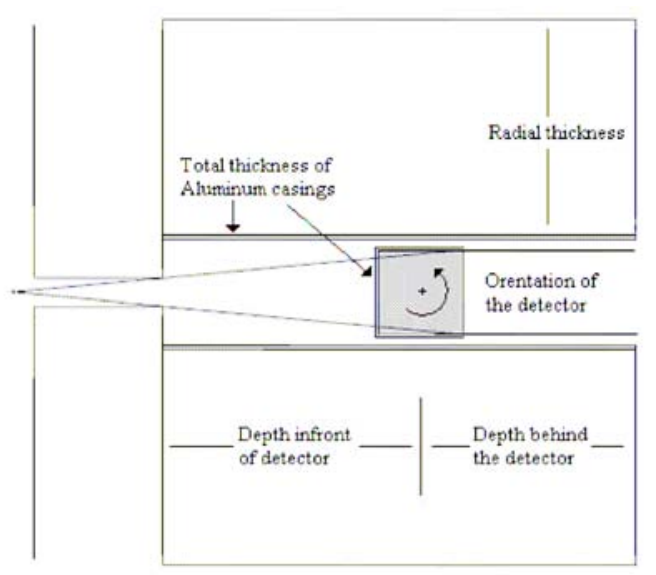

Figure 4: Detector geometry for the parameter optimization study.
A point source was placed $2.9 \mathrm{~cm}$ from the face of the SD having a tungsten collimator with a $5 \mathrm{~mm}$ aperture. Calculations only considered photons within 14 degrees of the $\mathrm{Z}$ axis to reduce the amount of computation time. The cut off energies for both photons and secondary electrons was $1 \mathrm{keV}$. In all cases the minimum deposited energy in the SD for cancellation was $10 \mathrm{keV}$. The error bars associated with the plotted values (from figures 5-9) represent one standard deviation. This standard deviation value is provided by the MCNP code and does not reflect uncertainty in the assumptions.

With in increasing radial thickness, the suppression factor increases asymptotically as is shown in Figure 5. For practical purposes nearly maximum suppression is achieved at an SD thickness of $100 \mathrm{~mm}$.

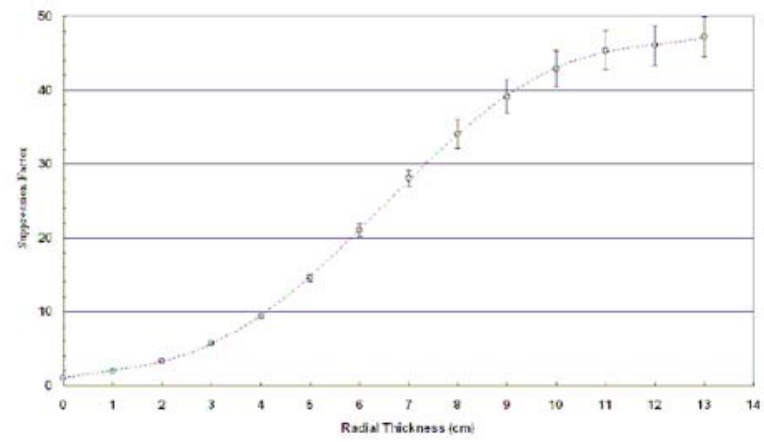

Figure 5 Illustrates the dependence of the suppression factor on radial thickness.

The depth of the PD from the face of the SD is the next parameter. The MCNP simulations indicated that optimal depth is about $50 \mathrm{~mm}$ as shown in Figure 6. This value seems reasonable considering the geometry of the system. The point source is located $29 \mathrm{~mm}$ from the face to the Compton guard. The internal diameter of the aperture is $5 \mathrm{~mm}$. A cone connecting the point source with the face of the detector intersects the outer edge of the detector at a distance of 58 $\mathrm{mm}$.

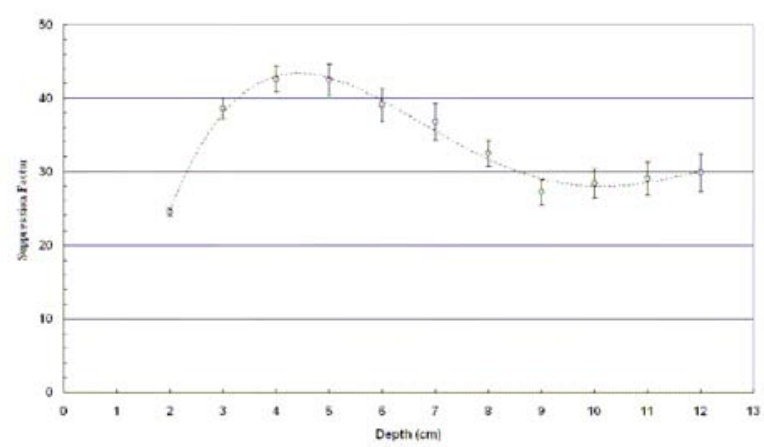

Figure 6 :Suppression factor as a function of the thickness of the SD in front of the PD.

The dependence of the suppression factor on the amount of SD behind the primary detector is displayed in Figure 7. The suppression factor approaches the maximum asymptotically 
with infinite SD thickness. Approximately 90 to $95 \%$ of the maximum suppression is realized at a SD thickness of $50 \mathrm{~mm}$ behind the PD.

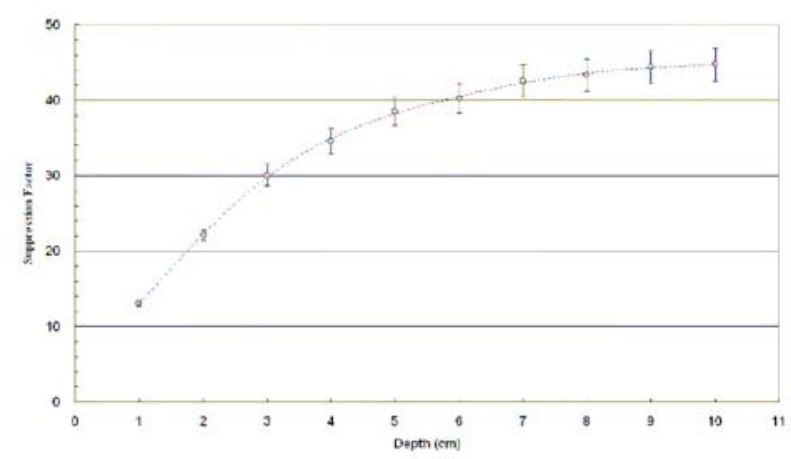

Figure 7: The dependence of the suppression factor on the amount of SD behind the primary detector.

When a scintillation crystal is used as the $\mathrm{PD}$, a light pipe or appropriately-sized photomultiplier is needed either to transfer or detect the scintillation light produced by gamma rays. The orientation of this detection path might have an influence on the efficiency of the Compton suppression due to the angular dependence of Compton scatter. To understand if there is an optimum orientation of the detection path, the angle at which the photomultipler was attached to the PD was explored. Figure 8 provides results of these calculations

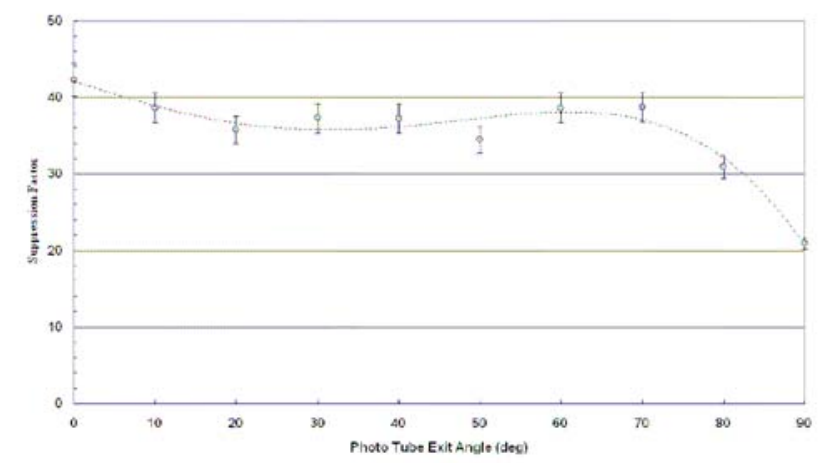

Figure 8: Suppression Factor vs. Phototube Exit Angle.

These results indicate that the angular orientation of the photomultipler does not strongly affect the suppression ratio. However, the typical $90^{\circ}$ orientation often employed for detectors of this type appears not to be the best orientation. Based on these calculations, the best orientation is to position the photomultiplier on the axis of symmetry and parallel to the gamma ray path.

Our reason for exploring these scintillation-based CSS is the fact that material thickness between the PD and SD negatively influences the magnitude of the suppression factor. However, the $\mathrm{LaCl}_{3}: \mathrm{Ce}$ scintillators are hydroscopic and are enclosed inside a windowed can. The wall thickness of the aluminum can influence the ultimate magnitude of the suppression factor. Further, if a metallic liner is placed between the PD and SD to make the SD light tight, the combination of these two pieces of material may significantly reduce the suppression efficiency of the corresponding detector. To investigate this dependence, a number of calculations were performed where all the properties of the detector were left unchanged while the thickness of the material between the PD and SD was varied. Figure 9 gives the results of these calculations.

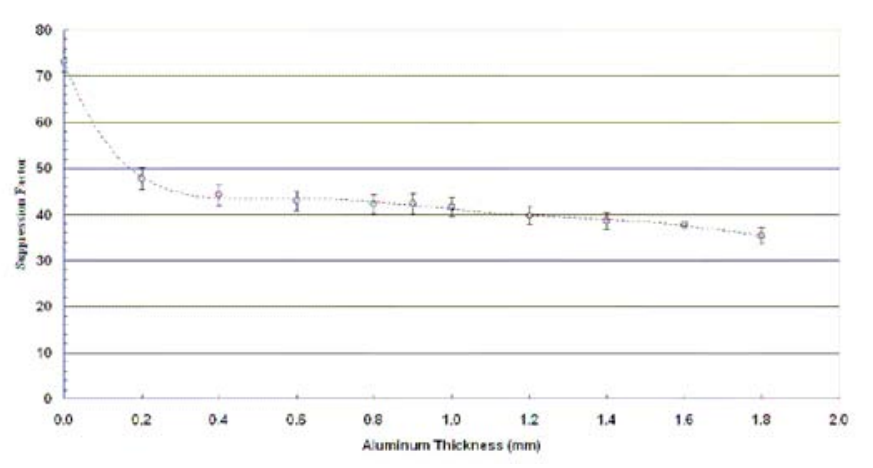

Figure 9: Suppression Factor versus Aluminum Thickness.

The results indicate that this material will ultimately limit the suppression efficiency of practical detector designs. This will be especially true for designs based on hydroscopic crystals that require some type of housing to protect the crystal.

\section{DISCUSSION}

In this paper we have revisited CSS parameter optimization using MCNP transport calculations. Because there are often a great number of assumptions and approximations made to model a CSS detector, we validated the MCNP transport model by comparing the model predictions of a ${ }^{137} \mathrm{Cs}$ source measured by two different types of CSS detectors with actual measurements obtained using two laboratory constructed units. The MCNP predicted spectra compare reasonably well with the experimental spectra. Further, the resulting suppression factors prepared from the model and experimental spectra are within in $20 \%$ of each other for our energy range of interest, of 100 to $300 \mathrm{keV}$. This agreement is sufficiently strong to suggest that we have a reasonable MCNP model for the CSS. The agreement between model and experiment results further supports the use of the model to predict optimum CSS parameters.

The parameter optimization studies suggest that for a $\mathrm{LaCl}_{3}: \mathrm{Ce}$ scintillator PD enclosed by cylindrical $\mathrm{NaI}(\mathrm{Tl}) \mathrm{SD}$ the optimum radial thickness for the SD is approximately 100 $\mathrm{mm}$. The optimum position of the PD within the NaI was centrally located along the axis of symmetry about $58 \mathrm{~mm}$ from the front surface of the SD and with about 50 to $60 \mathrm{~mm}$ of SD material behind the PD. CSS detectors base on scintillators require some path for the PD light to be detected. The orientation of this path was found not to be strongly sensitive to the angle between the axis of symmetry and light path. The conventional $90^{\circ}$ orientation significantly reduces 
the resulting suppression factor. This analysis indicates that a $0^{\circ}$ orientation relative to the axis of symmetry represents the optimum geometry.

Finally, to support our premise that reducing the amount of material between the PD and SD would have a significant impact on the Compton suppression, the thickness of the aluminum metal associated with the crystal aluminum enclosure was varied. Over the range of values examined, very little impact to the suppression factor was observed until the metal thickness decreased below $0.1 \mathrm{~mm}$. Between 0 and $0.1 \mathrm{~mm}$ there was a dramatic improvement in the resulting suppression factor. These results indicate that we need to find some way to remove the metal can that surrounds the $\mathrm{LaCl}_{3}: \mathrm{Ce}$ crystal. We are currently attempting to validate this conclusion experimentally.

\section{REFERENCES}

[1] E.L. Grigorescu, P. De Felice, A.C. Razdolescu, A. Luca, Appl. Rad. and Isotope 61 (2004) 191.

[2] J. Parus, J. Kierzek, W. Raab, D. Donohue, J. Radioanal. and Nuc. Chem. 258 (2003) 123.

[3] P. Peerani, P. Carbol, E. Hrnecek, M. Betti, Nucl. Instr. Meth. A 482 (2002) 42.

[4] E.V.D. Van Loef, P. Dorenbos, C.W.E. van Eijk, K.W. Krämer, H.N. Güdel, Nucl. Instr. Meth A 486 (2002) 254.

[5] C.P. Allier, E.V.D. van Loef, P. Dorenbos, R.W. Hollander, C.W.E.. van Eijk, K.W. Krämer, H.N. Güdel, Nucl. Instr. Meth. A 485 (2002) 547.

[6] L.L. Kiang, R.H. Tsou, W.J. Lin,S.C. Lin, G.C. Kiang, P.K. Teng, S.D. Li, Nucl. Instr. Meth. A 327 (1993) 427.

[7] H.M. Badran, T. Sharshar, Nucl. Instr. Meth. A 435 (1999) 423.

[8] L.L. Kiang, R.H. Tsou, J.H. Li, S.C. Lin, C.-Y. Lo, G.C. Kiang, P.K. Teng, Nucl. Instr. Meth. A 355 (1995) 434.

[9] Hendricks, J. S. McKinney, G. W. Waters, L. S. Roberts, T. L. Egdorf,H. W. Finch, J. P. Trellue, H. R.Pitcher, E. J. Mayo, D. R. Swinhoe, M. T.Tobin, S. J. Durkee, J. W. Gallmeier, F. X. David, J. C. Hamilton, W. B.Lebenhaft, J. MCNPX extensions version 2.5.0. Los Alamos National LaboratoryReport LA-UR-04-0570; 2004. 